\title{
Shaper-based infrared spectroscopy in a nonlinear Raman setup
}

\author{
Niklas Müller ${ }^{1}$, Lukas Brückner ${ }^{1}$, and Marcus Motzkus ${ }^{1, *}$ \\ ${ }^{1}$ Physikalisch-Chemisches Institut, Ruprecht-Karls Universität Heidelberg, 69120 Heidelberg, \\ Germany
}

\begin{abstract}
We present combined mid-infrared and nonlinear Raman spectroscopy in a single beam setup through the flexible control by sub $10 \mathrm{fs}$ pulse shaping. Hence tuneable $\left(>2000 \mathrm{~cm}^{-1}\right)$, narrowband $\left(<20 \mathrm{~cm}^{-1}\right)$ infrared light is generated.
\end{abstract}

\section{Introduction}

Nonlinear spectroscopic methods and in particular the combination of several nonlinear optical modalities in one setup, such as coherent Raman microscopy, second harmonic generation or two-photon fluorescence, have seen tremendous development over the recent years resulting in many different applications in both the material and life sciences [1]. In this contribution, we discuss the experimental realization of a novel concept for multimodal nonlinear microscopy that combines ultrashort, sub 10 fs laser pulses with pulse shapers (Fig.1a) and show in detail the successful implementation of adaptive spectral focusing (SF) schemes for Raman and mid-infrared (MIR) spectroscopy.

\section{Experimental Method}
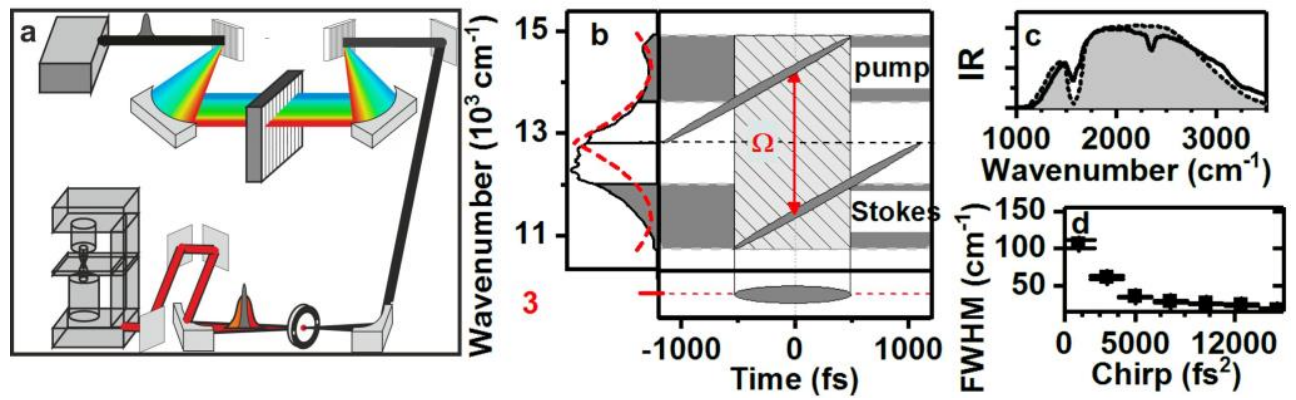

Fig. 1. (a) Combined setup for infrared and CARS micro spectroscopy. (b) Time frequency map of the spectral focusing concept. (c) Measured (solid line) and simulated (grey background) infrared spectra (d) Spectral infrared line width, displayed as the FWHM of the Gaussian response function.

\footnotetext{
*Corresponding author: marcus.motzkus@pci.uni-heidelberg.de
} 
The similarity in the infrared (IR) generation and the coherence excitation in the CARS process allows for the use of the same SF application. In the concept of SF [2], a phase of two spectrally separated chirps is imprinted on the laser pulses (Fig 1b, left panel).

The phase orders the frequencies in the two spectral regions to be linear in time (Fib 1b, middle panel). The difference of the frequency between the energetic higher (pump) and lower (Stokes) part is time independent, yielding narrowband excitation for difference frequency-based processes. In the IR generation (CARS spectroscopy), the instantaneous frequency difference (IFD), $\Omega$, between the pump and Stokes pulse generates a spectrally narrowband IR pulse (excitation of the coherence). In SF, both the excitation frequency and the spectral resolution can be changed by the IFD and chirp, respectively. For the IR generation, the tuning range (Fig. 1c) and the spectral width (Fig 1d) of the light was investigated [3]. The IR spectrum (Fig. 1c, solid line) generated in the $100 \mu \mathrm{m}$ thick $\mathrm{LiIO}_{3}$ with a chirp of $5000 \mathrm{fs}^{2}$ in the SF concept ranges from $1250 \mathrm{~cm}^{-1}$ up to $3500 \mathrm{~cm}^{-1}$. The measurements were validated by a simulation (grey background). For investigating the spectral width at a certain chirp, transmission spectra of a reference polystyrene film were recorded. The measured transmission spectra are the convolution of the molecular- and setup response functions. For the latter, a spectral Gaussian shape is assumed. The spectral width of the IR light, i.e. the FWHM of the Gaussian response function, decreases from more than $100 \mathrm{~cm}^{-1}$ at $1000 \mathrm{fs}^{2}$ to below $20 \mathrm{~cm}^{-1}$ at a chirp of $15000 \mathrm{fs}^{2}$ (Fig. 1d). In both the IR generation and the CARS spectroscopy, the pump and Stokes regions are limited by the desired frequency of the generated signal, yielding to some unusable parts of the spectrum. In the concept of tailored SF in CARS spectroscopy, this spectrally separated part of the spectrum can used as a temporal probe by imprinting a linear phase. Thus, the nonresonant background can be minimized by only detecting the anti-Stokes signal probed by the probe part. Moreover, the dynamics of the vibrational modes can be determined by scanning the probe delay relative to the coherence excitation [4].

The presented technique can be generally applicable in various setups containing different laser sources, using alternative shaping methods and nonlinear crystals. The setup we used (Fig. 1a) consists of a Ti:Sa oscillator, generating $800 \mathrm{~nm}$ centred sub $10 \mathrm{fs}$ laser pulses and a $4 \mathrm{f}$ pulse shaper setup. The shaped pulses are the bases for both the CARS and IR spectroscopy. For generating tuneable IR light, the shaped laser pulses are focused into a thin $\mathrm{LiIO}_{3}$-crystal for difference frequency generation. The anti-Stokes signal is generated in the sample within the microscope setup.

\section{Results}

For proving the versatile tuneable IR light source, a highly resolved transmission spectrum of a polystyrene film was recorded (Fig. 2a). The measured transmission at a chirp of $12500 \mathrm{~cm}^{-1}$ (solid line) follows the FTIR reference measurement (grey background) between $1250 \mathrm{~cm}^{-1}$ and $3250 \mathrm{~cm}^{-1}$ [3].

To demonstrate how tailored SF can enable time-resolved CARS as a viable technique, we measured the dynamics of the $\mathrm{C}-\mathrm{H}$ coherences of sunflower oil (depicted in Fig 2b). From the data depicted Fig 2b, a build-up and decay of the Raman lines at $2940 \mathrm{~cm}^{-1}$ and $3015 \mathrm{~cm}^{-1}$ relative to $2850 \mathrm{~cm}^{-1}$ is visible [4]. 

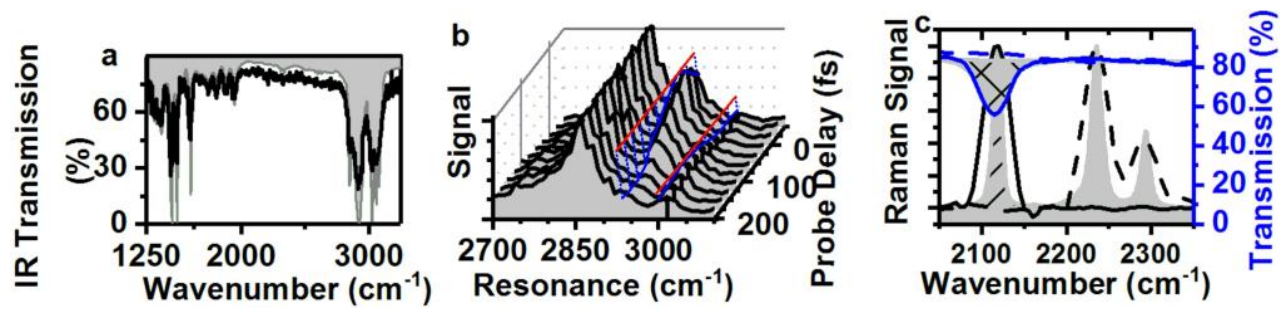

Fig. 2. (a) Polystyrene IR transmission measured in single beam SF setup (solid line) and FTIRreference measurement (grey background). (b) The time resolved CARS coherences of the $\mathrm{CH}$ resonances in sunflower oil indicates a build up at $3015 \mathrm{~cm}^{-1}$ and a decay at $2940 \mathrm{~cm}^{-1}$ relative to $2850 \mathrm{~cm}^{-1}$. (c) Raman signals (black lines) and IR transmissions (blue lines) for 1-Octyne (solid line) and 4-Octyne (dotted line). The corresponding references are given as the grey background for 1Octyne Raman (single stripes), 4-Octyne Raman (without stripes) and 1-Octyne IR transmission (crossed stripes).

For testing the combined IR and CARS setup, the rule of mutual exclusion for 1-Octyne and 4-Octyne was proven. Both molecules consist of a $\mathrm{C}$-C triple bonding, which is in the symmetry plane for 4-Octyne and thus makes it IR inactive. Fig. 2c displays the IR and Raman spectra for 1-Octyne (solid line) and 4-Octyne (dotted line), where the respective C$\mathrm{C}$ triple bond vibrations for both are located around $2120 \mathrm{~cm}^{-1}$ and $2250 \mathrm{~cm}^{-1}$. Whereas the vibrational Raman mode (black) is visible for both molecules, the IR transmission (blue) shows only a vibrational mode for 1-Octyne because of the IR inactivity of 4-Octyne. The data was recorded using a chirp of $7000 \mathrm{fs}^{2}$ and $9000 \mathrm{fs}^{2}$ for SF-based CARS and IR spectroscopy, respectively. The measurements are in good agreements with references (grey background in Fig. 2c) for 1-Octyne-Raman (single stripes), 4-Octyne Raman (without stripes) and 1-Octyne IR (crossed stripes), taken by a FTIR spectrometer and a Raman microscope [5].

\section{Summary}

A versatile approach using shaped, ultrashort laser pulses for combined IR and non-linear Raman spectroscopy in a single beam setup is presented. Hence, we have added to the previously reported nonlinear microscopy setup [4], which incorporated imaging resonant CARS, second harmonic generation and two photon induced excited state fluorescence, the capability of spectrally highly sensitive IR spectroscopy [3]. The spectral resolution in both cases is given by the concept of SF. Due to dependencies in the light generation process, the spectral resolution can be varied either for achieving the highest spectral resolution or matching the spectral width of a molecule yielding to the largest spatial contrast for imaging.

\section{References}

1. S. Yue, M. Slipchenko, Ji-Xin Cheng, Laser Photonics Rev., 5, 496 (2011)

2. G. Veitas, R. Danielius, JOSA B, 16, 1661 (1999).

3. L. Brückner, N. Müller, M. Motzkus, Opt. Lett, 43, 2054 (2018)

4. L. Brückner, T. Buckup, M. Motzkus, JOSA B, 33, 1482 (2016).

5. N. Müller, L. Brückner, M. Motzkus, APL Phot., 3, 092406 (2018) 